\title{
M. BENEDETTI \\ Étude d'une bande de roulement réservée aux autobus, établissement d'un modèle prévisionnel de simulation
}

Revue française d'automatique, d'informatique et de recherche opérationnelle. Recherche opérationnelle, tome 3, n V1 (1969), p. 3-16.

<http://www.numdam.org/item?id=RO_1969_3_1_3_0>

(C) AFCET, 1969, tous droits réservés.

L'accès aux archives de la revue « Revue française d'automatique, d'informatique et de recherche opérationnelle. Recherche opérationnelle » implique l'accord avec les conditions générales d'utilisation (http://www.numdam.org/ legal.php). Toute utilisation commerciale ou impression systématique est constitutive d'une infraction pénale. Toute copie ou impression de ce fichier doit contenir la présente mention de copyright.

\section{Numdam}


R.I.R.O.

(3e année, $\mathrm{N}^{\circ} \mathrm{V}-1,1969$, p. 3-16)

\title{
ETUDE D'UNE BANDE DE ROULEMENT RESERVEE AUX AUTOBUS ETABLISSEMENT D'UN MODELE PREVISIONNEL DE SIMULATION (1)
}

\author{
par M. BENEDETTI
}

\begin{abstract}
Résumé. - L'auteur étudie les différentes variables aléatoires définissant le parcautrs d'un autobus sur une voie. Il établit ensuite un modèle de simulation d'une bande de roulement réservée aux autobus, et en présente les avantages.

Le programme a été écrit en langage G.P.S.S. et testé sur l'ordinateur IBM 360-40
\end{abstract} de la R.A.T.P.

\section{INTRODUCTION}

Depuis quelques années, la Préfecture de Police a aménagé, sur certaines voies publiques, des bandes réservées à la circulation des autobus et dont l'accès est toléré aux taxis et aux véhicules prioritaires (ambulances, voitures de pompiers, etc.).

La création de telles bandes n'est possible que dans des artères suffisamment larges et ne se justifie que si ces dernières sont empruntées par de nombreuses lignes d'autobus.

L'existence d'une bande réservée apporte certaines gênes aux riverains (livraisons) ainsi qu'aux automobilistes (stationnement, possibilité de tourner à droite); de plus certaines modifications intervenant lors de la création d'une bande de roulement ont un caractère quasi irréversible (la signalisation par exemple). Il est donc souhaitable de pouvoir étudier, a priori, l'influence, tant sur les temps de parcours des autobus, que sur ceux des véhicules particuliers, que peut avoir une telle création. Un bilan pour la collectivité des gains et pertes de temps des usagers des autobus et des voitures particulières peut alors éclairer la décision à prendre.

L'auteur a mis au point un modèle prévisionnel (par une méthode de simulation), qui permet, à partir de comptages effectués dans la situation

(1) Résumé, rédigé par J. Dozias, de la thèse présentée par M. Benedetti pour obtenir le diplơme de statisticien, thèse effectuée sous les directives de G. Morlat, dans le cadre des recherches du groupe Prévision et Études de la Régie Autonome des Transports Parisiens. 
avant création de la bande, d'estimer les nouveaux temps de parcours des autobus, et qui donne la possibilité d'analyser l'influence de certains facteurs tels que la disparité des performances des matériels des différentes lignes, ou la présence de lignes exploitées avec des autobus à un agent (dont le service est plus lent). De plus ce modèle permet la comparaison de diverses politiques propres à l'usage d'une bande de roulement telles que, par exemple, le regroupement de plusieurs points d'arrêt.

Nota : Il n'a pas été tenu compte dans cette étude du droit des taxis et véhicules prioritaires à emprunter la bande, faute de comptages à ce sujet. Mais on peut observer, qu'en raison des possibilités d'accélération et de vitesse de ces véhicules, la gêne qu'ils causent aux autobus demeure en règle générale peu importante.

\section{DESCRIPTION D'UNE VOIE ET PARCOURS D'UN AUTOBUS SUR LA VOIE}

\section{II-1. Description d'une voie}

Toute voie parcourue par une ou plusieurs lignes d'autobus peut être décomposée en une succession de tronçons élémentaires et de points singuliers.

L'ensemble des points singuliers est composé :

- des points d'entrée et de sortie de chaque ligne sur la voie ;

- des points d'arrêt de chaque ligne ;

- des feux de signalisation.

Un tronçon élémentaire est une section de voie comprise entre deux points singuliers successifs.

\section{II-2. Parcours d'un autobus sur la voie}

Le parcours d'un autobus sur la voie est schématisé par les deux organigrammes suivants, dans le cas de la voie sans bande (Organigramme et dans le cas de la voie avec bande (Organigramme 2).

Il est supposé que tout autobus arrivant à un point d'arrêt de sa ligne ne peut échanger ses voyageurs que si aucun autobus ne s'y trouve.

On rappelle que les règles d'exploitation en vigueur à la R.A.T.P. interdisent, en principe, à deux autobus d'une même ligne de se doubler.

Les points singuliers susceptibles de modifier le temps de parcours d'un autobus sont :

- dans le cas de la voie sans bande :

- les points d'arrêt de sa ligne,

- les feux de signalisation,

- son point de sortie de la voie; 
- dans le cas de la voie avec bande :

- les points singuliers cités ci-dessus,

- les points d'arrêt des autres lignes.

Les modifications apportées à l'exploitation de lignes d'autobus par la création d'une bande de roulement résident essentiellement dans le

Organignamme 1

Parcours d'un autobus sur une yoie sans bande

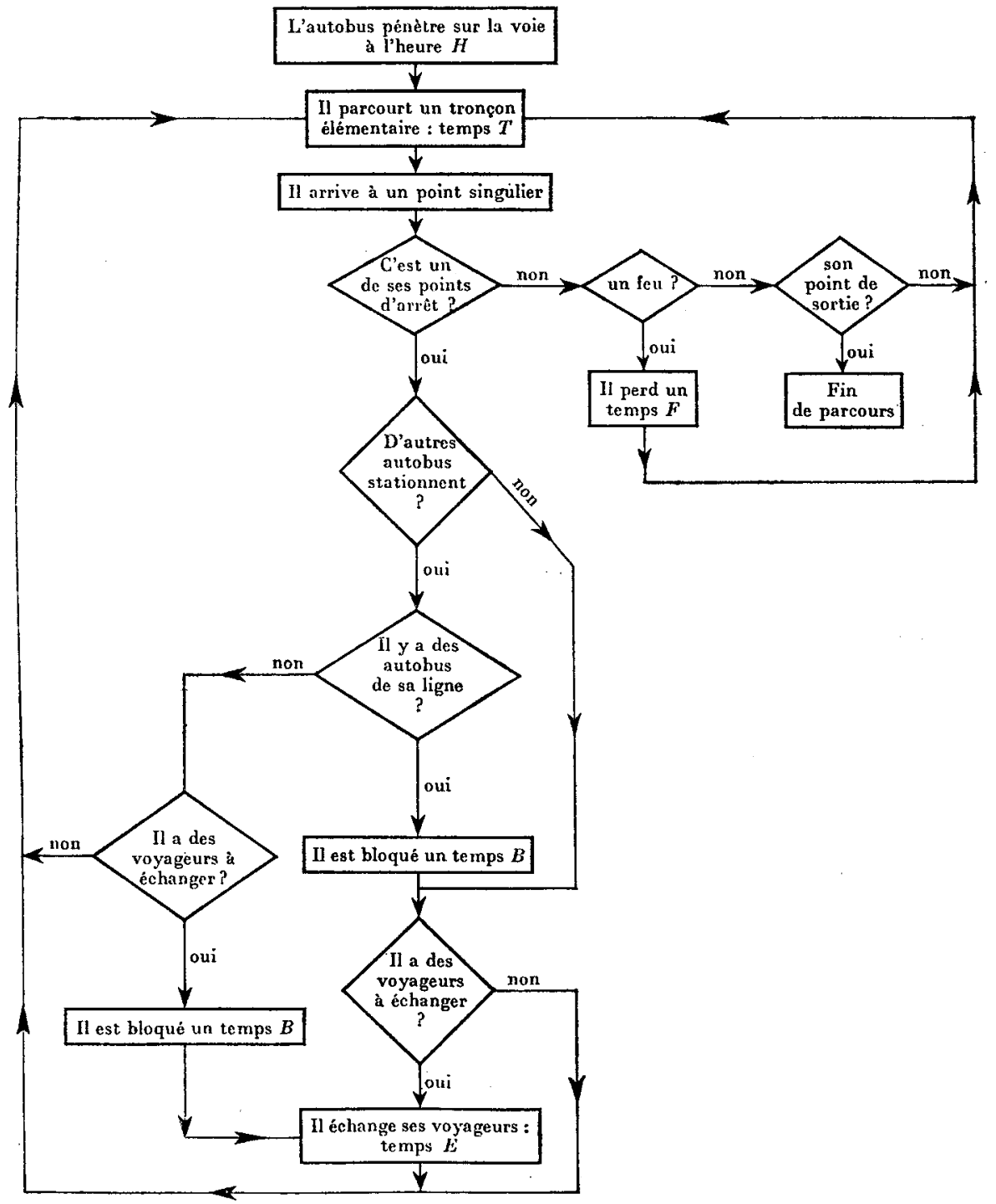


Organigramae 2

Parcours d'un autobus sur une voie avec bande

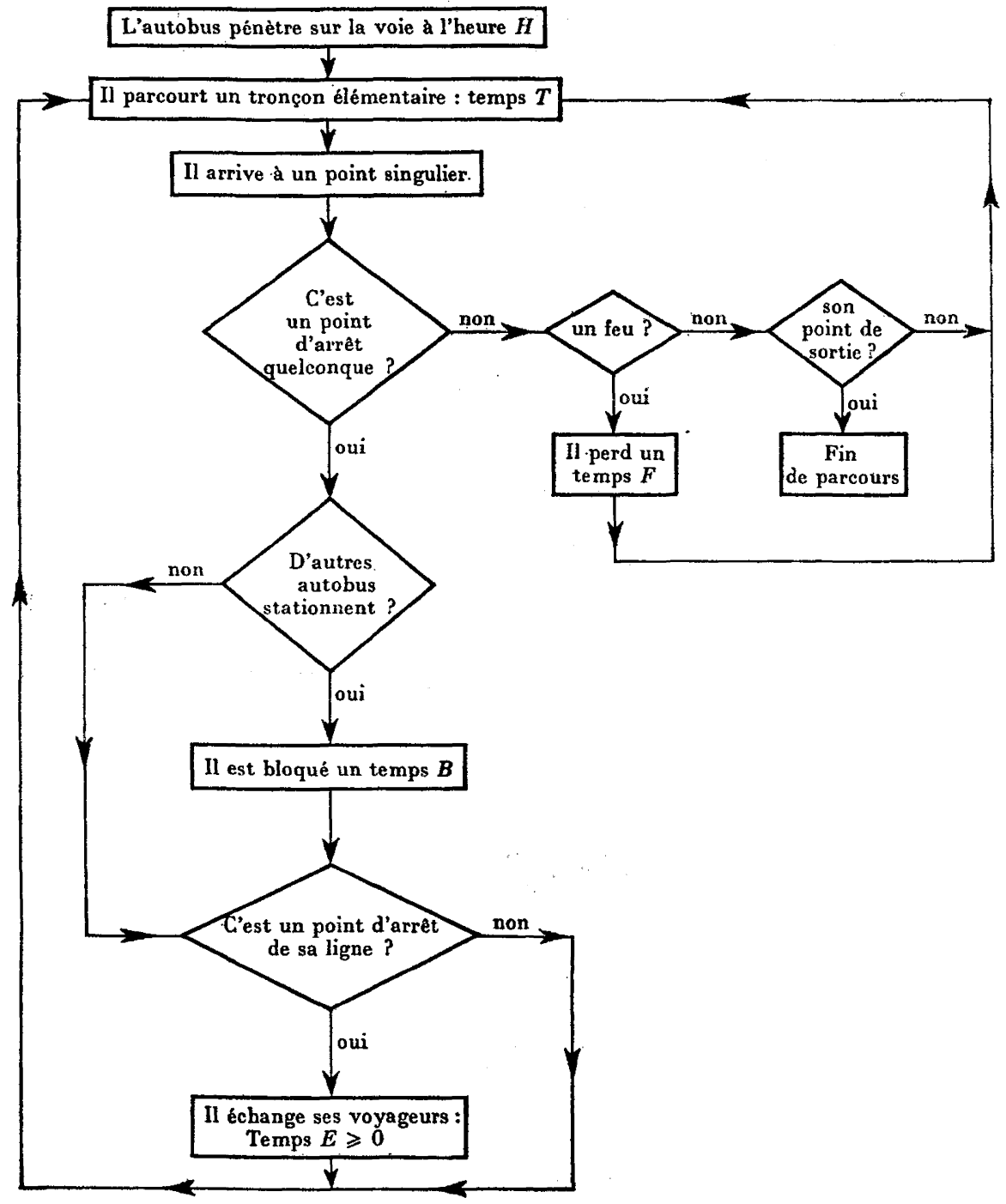

fait que les autobus roulent plus vite et à une vitesse plus régulière sur une bande, mais en revanche ils ne peuvent la quitter pour dépasser un autobus stationnant à un point d'arrêt qui n'est pas le leur. Ce sont principalement ces deux phénomènes qui sont mis en valeur dans la recherche du modèle. 


\section{ETUDE THEORIQUE DES VARIABLES DEFINISSANT LE PARCOURS D'UN AUTOBUS}

D'après ce qui précède, il apparaît que les variables aléatoires définissant le parcours d'un autobus sont :

1. - son heure d'arrivée $H$ à l'entrée de la voie étudiée,

2. - son temps de parcours $T$ sur un tronçon élémentaire,

3. - son temps d'attente $F$ aux feux de signalisation,

4. - son temps d'échange $E$ à ses points d'arrêt,

5. - son temps de blocage $B$ aux différents point d'arrêt.

Il faut introduire les lois de probabilité des variables $1,2,3,4$ dans le modèle recherché ; la variable $B$ se déduira, elle, de l'application du modèle.

\section{III-1. Processus d'arrivée des autobus d'une ligne à l'entrée de la voie}

L'heure $H$ est déterminée par le processus d'arrivée des autobus à l'entrée de la voie.

Les autobus partent du terminus suivant un processus périodique de période $t$.

Si $\theta_{k}$ désigne le temps de parcours du $k^{\text {leme }}$ autobus entre le terminus de départ et le point d'entrée sur la voie, et si $H_{k}$ désigne son heure d'arrivée sur la voie, alors,

$H_{k}=k t+\theta_{k}$ (le premier autobus partant à l'instant $t$ ).

Les variables $\theta_{k}$ ne sont pas indépendantes :

$\forall_{k} \theta_{k} \geqslant \theta_{k-1}-t$ (les autobus d'une même ligne ne se doublent pas).

Si $\theta_{k}^{\prime}$ désigne le temps qu'aurait mis l'autobus $k$ s'il n'avait pas été gêné par le précédent, on peut supposer que $\left(\theta_{k}^{\prime}\right)_{k \in K}$ est une suite de variables aléatoires indépendantes et de même loi.

Les temps de parcours réels $\theta_{k}$ se déduisent des $\theta_{k}^{\prime}$ de la façon suivante :

$$
\left\{\begin{array}{l}
\theta_{1}=\theta_{1}^{\prime} \\
\theta_{2}=\left\{\begin{array}{lll}
\theta_{2}^{\prime} & \text { si } & \theta_{2}^{\prime} \geqslant \theta_{1-t} \\
\theta_{1-\imath} & \text { si } & \theta_{2}^{\prime} \leqslant \theta_{1-t}
\end{array}\right. \\
\ldots \ldots \ldots \\
\theta_{k}=\left\{\begin{array}{lll}
\theta_{k}^{\prime} & \text { si } & \theta_{k}^{\prime} \geqslant \theta_{k-1-^{\prime}} \\
\theta_{k-1-^{\imath}} & \text { si } & \theta_{k}^{\prime} \leqslant \theta_{k-1-^{t}}
\end{array}\right.
\end{array}\right.
$$




\section{Remarque}

On considère que si un autobus est gêné pendant son trajet par l'autobus le précédant, ils arrivent tous deux au même instant à l'entrée de la voie. Ceci est en bonne conformité avec la réalité, car on constate que si un autobus en rattrape un autre pendant son trajet, il le suit généralement pendant toute la fin de son parcours.

On verra en III-2 que l'on peut considérer que $\theta_{k}^{\prime}$ obéit à une loi de Laplace-Gauss dont on estimera les paramètres $\left({ }^{1}\right)$.

\section{III-2. Temps de parcours sur un tronçon élémentaire (voie sans bande)}

Ce temps de parcours $T$ peut s'écrire :

$$
T=t_{0}+\sum_{j=1}^{N} T_{j}
$$

où $t_{0}$ désigne le temps minimum nécessaire à parcourir le tronçon et où $T_{j}$ désigne le retard élémentaire pris par l'autobus à la suite d'un événement l'ayant obligé à s'arrêter ou à freiner. $N$ est le nombre de ces retards élémentaires (on considère ici des autobus ne rattrapant pas l'autobus précédent).

En faisant les hypothèses suivantes :

- les événements décrits ci-dessus sont mutuellement indépendants,

- ils peuvent se produire de façon uniforme sur tout le tronçon,

- la probabilité qu'il ne s'en produise aucun sur une distance quelconque est différente de 0 et de 1 , le processus étudié est de Poisson et uniforme.

Le nombre $N$ de retards élémentaires accumulés par l'autobus sur un tronçon de longueur $l$ obéit à une loi de Poisson de moyenne $\lambda=k l$, si $k$ est la densité du processus.

\section{III-2.1. Etude de $\boldsymbol{T}$ sous les deux hypothèses (1 et 2 ou 1 et $2^{\prime}$ ) :}

(1) les variables aléatoires $T_{j}$ sont mutuellement indépendantes, continues, positives ou nulles et de même loi.

(2) $N$ appartient à une loi de Poisson de moyenne $\lambda$.

(2') $N$ est constant $N=\lambda$.

Soient $\varphi(u)$ et $\psi(u)$ les première et deuxième fonctions caractéristiq es (f.c.) de $T_{j}$. On désigne par $\Phi(u)$ et $\Psi(u)$ les première et deuxième f. $\operatorname{de} \theta=T-t_{0}$.

(1) L'auteur montre de plus que l'on peut définir la suite des $\theta_{k}$ comme une suite de Markov homogène. Si ce résultat est intéressant d'un point de vue théorique, il ne permet pas de simplifier l'étude du modèle de simulation recherché. 
Si $N$ obéit à une loi de Poisson, la variable aléatoire $\theta$ est continue pour toute valeur strictement positive mais elle prend la valeur 0 avec une probabilité non nulle :

$$
\begin{aligned}
P(\theta= & 0)=P(N=0)=\mathrm{e}^{-\lambda} \\
\Phi(u) & =E\left(\mathrm{e}^{i u \sum_{1}^{N} T_{j}}\right) \\
& =E\left[E^{N=n}\left(\mathrm{e}^{i u \sum_{1}^{N} T_{j}}\right)\right] \\
& =E\left[(\varphi(u))^{N}\right] \quad \text { cf. (1) }
\end{aligned}
$$

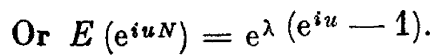

D'où :

$$
\begin{aligned}
& \Phi_{T-t_{0}}(u)=\mathrm{e}^{\lambda(\varphi(u)-1)} \\
& \Psi_{T-t_{0}}(u)=\lambda(\varphi(u)-1)
\end{aligned}
$$

Si $m_{q}$ et $\mu_{q}$ sont les moments non centré et centré d'ordre $q$ de $T_{j}$, alors :

$$
\varphi(u)=1+(i u) m_{1}+(i u)^{2} \frac{m_{2}}{2 !}+\ldots
$$

Done :

$$
\Psi_{T-t_{0}}(u)=\lambda m_{1}(i u)+\lambda m_{2} \frac{(i u)^{2}}{2 !}+\ldots
$$

et

$$
\begin{aligned}
& E(T)=t_{0}+\lambda m_{1} \\
& V(T)=\lambda m_{2}
\end{aligned}
$$

De plus quand $\lambda \rightarrow \infty$ la loi de $T$ converge $\left(\right.$ en $\left.\frac{1}{\sqrt{\lambda}}\right)$ vers une loi normale.

En effet :

$$
\begin{aligned}
\Psi_{\frac{T-E(T)}{\sigma(T)}}(u) & =-i u \frac{E(T)}{\sigma(T)}+\Psi_{T}\left(\frac{u}{\sigma(T)}\right) \\
& =-\frac{u^{2}}{2 !}+\frac{(i u)^{3}}{3 !}-\frac{m_{3}}{m_{2}^{\frac{3}{2}}} \frac{1}{\sqrt{\lambda}}+\varepsilon(\lambda)
\end{aligned}
$$

$(\varepsilon(\lambda) \rightarrow 0$ quand $\lambda \rightarrow+\infty)$. 
Dans le cas particulier où $N$ est constant $(N=\lambda=k l)$,

$$
\begin{gathered}
\Psi_{T-t_{0}}(u)=\lambda \log \varphi(u)=\lambda \psi(u) \\
\Psi_{T-t_{0}}(u)=\lambda i u m_{1}+\lambda \frac{(i u)^{2}}{2 !} \mu_{2}+\lambda \frac{(i u)^{3}}{3 !} \mu_{3}+\ldots \\
\begin{array}{l}
E(T)=t_{0}+\lambda m_{1} \\
V(T)=\lambda \mu_{2}
\end{array}
\end{gathered}
$$

De plus :

$$
\Psi_{\frac{T-E(T)}{\sigma(T)}}(u)=-\frac{u^{2}}{2 !}+\frac{(i u)^{3}}{3 !} \frac{\mu^{3}}{\mu_{2} \frac{3}{2}} \frac{1}{\sqrt{\lambda}}+\varepsilon(\lambda)
$$

$(\varepsilon(\lambda) \rightarrow 0$ quand $\lambda \rightarrow \infty)$

Donc si la loi de $T_{j}$ est symétrique $\left(\mu_{3}=0\right)$, la convergence de la loi de $T$ vers la loi normale quand $\lambda \rightarrow \infty$ est beaucoup plus rapide dans le

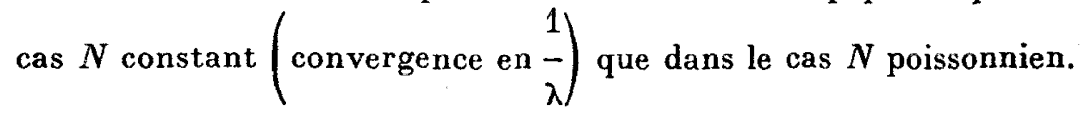

\section{III-2.2. Hypothèses sur la loi des $T_{j}$}

Le retard dû à un événement obligeant l'autobus à s'arrêter ou à freiner a, par sa nature élémentaire, une probabilité forte d'être faible, et une probabilité faible d'être grand.

Ceci permet de supposer que la loi de $T_{j}$ est une loi $\gamma$ de paramètres $p$ et $c: c T_{j} \in \gamma(p)$.

Alors,

$$
\begin{aligned}
& m_{1}=E\left(T_{j}\right)=\frac{p}{c} \\
& \mu_{2}=V\left(T_{j}\right)=\frac{p}{c^{2}}
\end{aligned}
$$$$
\text { et } \quad \varphi_{T_{j}}(u)=\left(1-\frac{i u}{c}\right)^{-p}
$$ 
Si $N$ obéit à une loi de Poisson de moyenne $k l$, alors d'après (3) et (4) :

$$
\begin{gathered}
\Psi_{T-t_{0}}(u)=k l\left[\left(1-\frac{i u}{c}\right)^{-p}-1\right] \\
\Psi_{\frac{T-E(T)}{\sigma(T)}}(u)=-\frac{u^{2}}{2}+\frac{p+2}{\sqrt{p(p+1)}} \frac{(i u)^{3}}{3 ! \sqrt{k l}}+\varepsilon(k l) \\
E(T)=t_{0}+k l \frac{p}{c} \\
V(T)=k l \frac{p(p+1)}{c^{2}}
\end{gathered}
$$

Si $N$ est constant $(N=k l)$, d'après $(5)$ et $(6)$ :

$$
\begin{gathered}
\Psi_{T-t_{0}}(u)=-k l p \log \left(1-\frac{i u}{c}\right) \quad\left(c\left(T-t_{0}\right) \in \gamma\left(k l_{p}\right)\right) \\
\Psi_{\frac{T-E(T)}{\sigma(T)}}^{s}(u)=-\frac{u^{2}}{2}+\frac{(i u)^{3}}{3 \sqrt{k l_{p}}}+\varepsilon(k l) \\
E(T)=t_{0}+k l \frac{p}{c} \\
V(T)=k l \cdot \frac{p}{c^{2}}
\end{gathered}
$$

\section{REMARQUES}

$1^{\circ}$ La convergence de la loi de $T$ vers la loi normale quand $k l \rightarrow \infty$ est plus rapide dans le cas où $N$ est constant car

$$
\frac{p+2}{6 \sqrt{p(p+1)}}>\frac{1}{3 \sqrt{p}} \forall p>0
$$

$2^{\circ}$ Quand $N$ est poissonnien avec $k l \rightarrow \infty, p \rightarrow 0$ mais $k l_{p}=r$ alors $T-t_{0}$ converge vers la loi $\gamma$ de paramètres $r=k l p$ et $c$.

En effet :

Si $p \rightarrow 0$ alors

$$
\begin{aligned}
\Psi_{T-t 0}(u) & =k l\left[\left(1-\frac{i u}{c}\right)^{-p}-1\right] \\
& =\frac{r}{p}\left[\mathrm{e}^{-p \log \left(1-\frac{i u}{c}\right)}-1\right]
\end{aligned}
$$

$$
\Psi_{T-\text { od }}(u)=-r \log \left(1-\frac{i u}{c}\right)+\varepsilon(p) \quad \text { avec } \quad \varepsilon(p) \rightarrow 0
$$


Soit en résumé :

$$
T=t_{0}+\sum_{1}^{N} T_{j} ; \quad N \in \mathfrak{T}(k l) ; \quad T_{j} \in \gamma(p, c)
$$

\begin{tabular}{|c|c|c|}
\hline \multicolumn{2}{|l|}{ HYPOTHESES } & LOI DE $T-t_{0}$ \\
\hline $\begin{array}{l}\text { Circulation embouteillée } \\
\text { ou } \\
\text { tronģon de grande longueur (1) }\end{array}$ & $k l$ grand & $\begin{array}{c}L G(m, \sigma) \\
m=\frac{k l p}{c} \quad \sigma^{2}=\frac{k l p(p+1)}{c^{2}}\end{array}$ \\
\hline Circulation fluide & $\begin{array}{l}k l \text { grand } \\
p \text { petit }\end{array}$ & $\begin{array}{c}\gamma(k l p, c) \\
m=\frac{k l p}{c} \quad \sigma^{2}=\frac{k l p}{c^{2}}\end{array}$ \\
\hline
\end{tabular}

III-2.3. Loi utilisée dans le modèle et détermination de ses paramètres (tronçon sans bande)

On détermine un échantillon des temps de parcours $T$ correspondant aux autobus qui n'ont manifestement pas été gênés par un autobus les précédant.

Connaissant la vitesse maximum (dépendant des performances du matériel) on détermine $t_{0}$, et l'on teste l'appartenance de $X=T-t_{0}$ à une loi $\gamma\left(r=k^{\prime} l, c\right)$.

Sur un tronģon $i, r_{i}$ et $c_{i}$ sont estimés par

$$
\begin{aligned}
\widehat{ }_{1} \widehat{r}_{i} & =\frac{{ }_{i} \bar{X}^{2}}{{ }_{i} s_{X}^{2}} \\
\widehat{c}_{i} & =\frac{{ }_{i} \bar{X}}{{ }_{i} S_{X}^{2}}
\end{aligned}
$$

Si la circulation est homogène sur toute la voie, on pourra obtenir des estimations globales de $k^{\prime}$ et $c$ à l'aide d'échantillons sur $n$ tronçons de longueur $l_{i}(i=1, n)$ par

$$
\begin{aligned}
\hat{k}^{\prime} & =\frac{1}{n} \sum_{i=1}^{n} \frac{{ }_{1} \frac{\hat{r_{i}}}{l_{i}}}{{ }_{2} \widehat{r}_{i}}=\widehat{k}^{\prime} l_{i} \\
\widehat{c} & =\frac{\bar{X}}{s_{X}^{2}}
\end{aligned}
$$


où $X$ désigne l'échantillon unique obtenu en rassemblant les $n$ échantillons ${ }_{i} X$.

Ces estimations ${ }_{2} \widehat{r}_{i}$ présentent l'avantage d'être proportionnelles aux longueurs $l_{i}$.

On a :

$$
\begin{aligned}
& E\left({ }_{2} \widehat{r}_{i}\right)=\frac{l_{i}}{n} \sum_{i=1}^{n} \frac{E\left({ }_{1} \widehat{r}_{i}\right)}{l_{i}} \\
& V\left({ }_{2} \widehat{r}_{i}\right)=\frac{l_{i}{ }^{2}}{n^{2}} \sum_{i=1}^{n} \frac{V\left({ }_{1} \widehat{r}_{i}\right)}{l_{i}{ }^{2}}
\end{aligned}
$$

Pour le tronçon $i_{0}$ tel que :

$$
\frac{V\left(\widehat{r}_{i_{0}}\right)}{l_{i_{0}}^{2}}=\operatorname{Sup}_{i=1, n} \frac{V\left(\widehat{r}_{1}\right)}{l_{i}^{2}}
$$

La variance de ${ }_{2} r_{i_{0}}$ est plus faible que celle de ${ }_{1} r_{i_{0}}$ :

$$
V\left({ }_{2} \widehat{r}_{i_{0}}\right) \leqslant \frac{V\left(\widehat{r}_{i_{0}}\right)}{n}
$$

\section{III-3. Temps d'attente aux feux de signalisation}

En décomposant le cycle d'un feu en :

- un temps $\lambda$ où il est vert (ou orange),

- un temps $\mu$ où il est rouge,

et en supposant que les autobus arrivent au hasard à l'intérieur d'un cycle, l'auteur montre que le temps moyen passé à un feu de signalisation est :

$$
E(F)=\frac{\mu^{2}}{2(\lambda+\mu)}
$$

alors que l'écart-type est :

$$
\sigma(F)=\frac{\mu}{2(\lambda+\mu)} \sqrt{\frac{\mu(4 \lambda+\mu)}{3}}
$$

\section{III-4. Temps d'échange des voyageurs aux points d'arrêt}

Le temps d'échange est le temps d'immobilisation nécessaire à un autobus pour faire monter et descendre ses voyageurs à un point d'arrêt. Ce temps est essentiellement imposé par le nombre de voyageurs échangés. La nature du modèle du temps d'échange dépend du matériel utilisé. 
Ainsi, pour les autobus à plateforme où la montée s'effectue après la escente, le modèle est de la forme :

$$
E=a+b N_{m}+c N_{d}+\varepsilon
$$

où $a, b, c$ sont les coefficients de la régression de $E$ sur $N_{m}$ et $N_{d}, N_{m}$ et $N_{d}$ sont respectivement les nombres de voyageurs montés et descendus, et où $\varepsilon$ est un résidu aléatoire suivant une loi normale de moyenne nulle.

Pour le matériel moderne, la montée et la descente s'effectuent simultanément, et le type de modèle est de la forme :

$$
E=f\left(N_{m}, N_{d}\right)+\varepsilon \quad \varepsilon \in L G(0, \sigma)
$$

la fonction $f$ pouvant s'écrire, par exemple,

$$
f\left(N_{m}, N_{d}\right)=a+b \operatorname{Sup}\left(p N_{m}, N_{d}\right) .
$$

La loi de $E$ liée par $N_{m}$ et $N_{d}$ est alors une loi de Laplace-Gauss de moyenne $f\left(N_{m}, N_{\mathrm{d}}\right)$ et d'écart-type $\sigma$.

Il est donc nécessaire, dans le cas général, de connaître les lois de probabilité de $N_{m}$ et $N_{\mathrm{d}}$. On a pu se contenter ici d'un modèle simplifié :

Pour chaque ligne, on définit la probabilité $p$ qu'a un autobus de ne pas s'arrêter à un point d'arrêt donné (c'est-à-dire de n'avoir aucun voyageur à échanger). Cette probabilité peut être considérée comme constante pendant la période étudiée (heure de pointe, où le trafic-voyageurs varie peu).

On suppose que la loi du temps d'échange conditionnée par le fait qu'il n'est pas nul est une loi de Laplace-Gauss (tronquée). Une condition suffisante mais non nécessaire pour qu'il en soit ainsi est que les nombres de voyageurs montés et descendus puissent être considérés comme constants sur la période étudiée.

On vérifie par le test classique du $\chi^{2}$ que l'hypothèse d'une loi de Laplace-Gauss est justifiée et l'on estime ses paramètres.

\section{MODELE PREVISIONNEL DE SIMULATION}

On peut à l'aide des variables étudiées en III définir un modèle théorique de la circulation des autobus sur une bande de roulement, mais, même avec des hypothèses simplificatrices, la résolution analytique du modèle est extrêmement complexe : elle s'est avérée infructueuse. Une méthode de simulation est alors toute indiquée et présente, de plus, l'avantage de serrer la réalité de plus près.

Il s'agit évidemment d'une simulation d'un phénomène aléatoire (parcours des autobus sur une voie) à partir d'échantillons fictifs constitués à l'aide de nombres au hasard. 
L'étude faite en III a permis l'écriture du modèle, écriture et mise au point ayant été effectuées en six semaines environ.

Le modèle étudié est général puisqu'il se rapporte à une bande de roulement interrompue en un ou plusieurs endroits.

Le programme se compose des sous-programmes suivants:

- génération des autobus à l'entrée de la bande,

- parcours sur un tronçon élémentaire (voie sans bande, et voie avec bande), bande),

- stationnement à un point d'arrêt (voie sans bande et voie avec

- arrivée à un feu de signalisation,

- fin de parcours.

Le parcours des autobus sur un tronçon élémentaire d'une voie avec bande est effectué en prenant une vitesse de croisière constante et en différenciant le cas où la vitesse de départ est nulle de celui où elle est non nulle.

Pour tout parcours d'un tronçon élémentaire un test est fait de façon à empêcher deux autobus d'une même ligne (ou deux autobus quelconques) de se doubler sur une voie sans bande (resp. sur une voie avec bande).

De même un test est nécessaire pour imposer à tout autobus arrivant à un point d'arrêt de n'échanger ses voyageurs que si aucun autobus ne s'y trouve.

Bien que connaissant la loi du temps $F$ passé à un feu de signalisation, la réalité a été reproduite en créant des unités fictives qui occupent les feux durant le temps où ils sont rouges. L'autobus ne peut alors passer que si le feu est "libre ».

La simulation a été effectuée à l'aide du langage G.P.S.S. (General Purpose System Simulation) dont le principe est le suivant :

des transactions, que l'on peut créer et détruire, circulent en séquence dans différents blocs, le passage dans un bloc déclenchant un sousprogramme qui se déroule automatiquement.

Ce modèle a été testé sur le cas de la bande interrompue de la rue de Rivoli. Des comptages faits après création de la bande de roulement ont permis d'ajuster certains paramètres, tels que la vitesse de croisière d'un autobus sur un tronçon élémentaire d'une voie avec bande, et de s'assurer de la validité du modèle.

On a ainsi pu vérifier que la création de cette bande de roulement avait entraîné, pour la ligne 67 , ligne parcourant entièrement la voie étudiée, un gain de temps moyen, entre 15 heures et 17 heures, de 170 secondes environ par autobus. Ce gain aurait été de 215 secondes, si la bande n'avait pas été interrompue. 


\section{CONCLUSION}

L'utilisation du modèle proposé nécessite le recueil de nombreuses données et leur traitement statistique. Si ce travail préparatoire est assez lourd, le modèle de simulation présente, en revanche, de nombreux avantages :

- il permet de reproduire la réalité avec beaucoup de précision, puisque l'on peut notamment représenter la gêne mutuelle des autobus en les empêchant de se doubler, de même que reconstituer le fonctionnement des feux de signalisation;

- il se prête facilement à des modifications l'adaptant à des circonstances particulières, ce qui donne la possibilité de comparer diverses politiques et par approches successives, permet de déterminer le système d'exploitation optimal d'une bande de roulement. 